\title{
Gravitational flux tubes
}

\author{
V. Dzhunushaliev ${ }^{\mathrm{a}, \mathrm{b}, 1}$, U. Kasper ${ }^{\mathrm{c}, 2}$, D. Singleton ${ }^{\mathrm{d}, 3}$ \\ ${ }^{\text {a } U n i v e r s i t a ̈ t ~ P o t s d a m, ~ I n s t i t u t ~ f u ̈ r ~ M a t h e m a t i k, ~ 14469, ~ P o t s d a m, ~ G e r m a n y ~}$ \\ b Theoretical Physics Department, KSNU, 720024, Bishkek, Kyrgyzstan \\ ${ }^{\mathrm{c}}$ Trojanstr. 7, D-12437 Berlin, Germany \\ ${ }^{\mathrm{d}}$ Department of Physics CSU Fresno, 2345 East San Ramon Ave. M / S 37 Fresno, CA 93740-8031, USA
}

Received 8 November 1999; accepted 7 March 2000

Editor: R. Gatto

\begin{abstract}
By studying multidimensional Kaluza-Klein theories, or gravity plus U(1) or SU(2) gauge fields it is shown that these theories possess similar flux tube solutions. The gauge field which fills the tube geometry of these solutions leads to a comparison with the flux tube structures in QCD. These solutions also carry a "magnetic" charge, $Q$, which for the SU(2) Einstein-Yang-Mills (EYM) system exhibits a dual relationship with the Yang-Mills gauge coupling, $g,(Q=1 / g)$. As $Q \rightarrow 0$ or $Q \rightarrow \infty, g \rightarrow \infty$ or $g \rightarrow 0$ respectively. Thus within this classical EYM field theory we find solutions which have features - flux tubes, magnetic charges, large value of the gauge coupling - that are similar to the key ingredients of confinement in QCD. (C) 2000 Elsevier Science B.V. All rights reserved.
\end{abstract}

PACS: $04.50 .+\mathrm{h} ; 04.20 . \mathrm{Jb} ; 12.90 .+\mathrm{b}$

Keywords: Gravitational flux tube; Confinement

\section{Introduction}

In this article we consider space-times of dimension $D=4,5$, or 7 . In the $D=4$ case we also include gauge fields, while in the higher dimensional cases the gauge fields arise naturally from the extra dimensions in the Kaluza-Klein way. The external 4-dimensional (4D) space-times that we consider have the topological structure $R^{1} \times R^{1} \times D^{2}$ or $R^{1}$ $\times R^{1} \times S^{2}$ (where $D^{2}$ is topologically the 2-dimen-

\footnotetext{
${ }^{1}$ E-mail: dzhun@rz.uni-potsdam

${ }^{2}$ E-mail: ukasper@rz.uni-potsdam.de

${ }^{3}$ E-mail: das3y@zimmer.csufresno.edu
}

sional (2D) disk and $\boldsymbol{S}^{\mathbf{2}}$ the 2D sphere). In both cases we take the external 3-dimensional (3D) spaces to have a tube structure so that locally the metric appears as

$d s_{3}^{2}=d r^{2}+a^{2}\left(d \theta^{2}+\sin ^{2}(\theta) d \phi^{2}\right)$

with $a$ being a constant. For the first topology the coordinates take the range

$-\infty<r+<\infty, \quad 0<\theta<\pi, \quad-\pi / 2<\phi<\pi / 2$

while in the second case the $\phi$ range is altered to

$-\pi<\phi<\pi$

These ranges are in accordance with the usual conventions for the $2 \mathrm{D}$ disk and $2 \mathrm{D}$ sphere respectively. 
These two cases differ in the identification of the boundary points.

The metric of the external 4D space-time is then $d s^{2}=e^{2 \nu(r)} d t^{2}-d s_{3}^{2}$

This type of metric was considered in Ref. [1] with the source term in Einstein's equations being an electric field. The ansatz function, $e^{\nu}$, for the flux tube solution of this 4D Einstein-Maxwell system is given by

$e^{\nu}=c_{1} e^{r / a}+c_{2} e^{-r / a}$

with $c_{1}, c_{2}$ being constants of integration.

In Ref. [2] a magnetic field was included in addition to the electric field. The form of these fields was given by

$F_{10}=\rho^{1 / 2} \cos \alpha e^{\nu}, \quad F_{23}=a^{2} \rho^{1 / 2} \sin \alpha \sin ^{2}(\theta)$

where $\alpha$ is an arbitrary constant and $\rho$ satisfies

$\rho=\frac{8 \pi}{\kappa a^{2}}$

$\kappa$ is Einstein's constant. We use units such that $c=1$ and $\hbar=1$ so that $\kappa$ has dimension length ${ }^{2}$ and the electromagnetic charges are dimensionless. In this case $c_{1}, c_{2}$ are required to take values such that $e^{\nu(r)}=\cosh (r / a)$ in Eq. (5). With this the metric in Eq. (4) becomes

$$
\begin{aligned}
d s^{2}= & \cosh ^{2}(r / a) d t^{2}-d r^{2} \\
& -a^{2}\left(d \theta^{2}+\sin ^{2}(\theta) d \phi^{2}\right)
\end{aligned}
$$

It is possible to chose coordinates such that $F_{01}$ and $F_{23}$ are constant. In Ref. [3], a metric of this type was considered for the construction of compactified phases in 4D space-times ${ }^{4}$.

Having briefly introduced the flux tube solution for the 4D Einstein-Maxwell system, we will turn in the following sections to examining $5 \mathrm{D}$ and $7 \mathrm{D}$ Kaluza-Klein metrics, and 4D Einstein-Yang-Mills systems all of which exhibit similar flux tube solutions (i.e. metrics whose 3D space is like that in Eq. (1), and with gauge fields running down the length

\footnotetext{
${ }^{4}$ We note that the components of the electromagnetic field (2b) in Ref. [3] are not those belonging to the coordinate system chosen in (2a).)
}

of the tube structure). We will also point out some important differences between these solution (e.g. the $4 \mathrm{D}$ space-time of the 7D solution with the internal group of $\mathrm{SU}(2)$ is not a solution of the 4D Einstein-Yang-Mills equations with gauge group $\mathrm{SU}(2))$.

The "flux tube" solutions that are presented here bear some resemblance to the flux tubes which occur in type-II superconductors and also in quantum chromodynamics (QCD). In QCD a tube of constant chromodynamic fields is thought to form between quark-antiquark pairs. In the gravitational solutions presented the 3D hypersurfaces, $t=$ const, are tubes filled with some gauge field. The flux tubes in QCD are thought to be closely connected with the confinement mechanism. The similarity between the QCD flux tubes and the gravitational flux tubes might indicate some analogous classical confinement mechanism for gravity theories (i.e. these solution might indicate a gravitational "confinement" of two (chromo)electrical and/or (chromo) magnetic charges via a flux tube stretched between them).

The main goals of our paper are to make the case:

- that the flux tube solutions are typical solutions of gravitational theories in the presence of gauge fields.

- that these gravitational solutions may indicate some link with the flux tubes of QCD. Further, for the 4D Einstein-Yang-Mills (EYM) system we find a very strong similarity between the structure of the solutions and some key features of the standard mechanism of confinement of color charge.

\section{5D Kaluza-Klein theory}

In this section we will look at a 5D Kaluza-Klein version of the 4D solution given in the introduction, and compare the two cases. The general 5D Kaluza-Klein metric is given by

$d s_{5}^{2}=g_{\mu \nu} d x^{\mu} d x^{\nu}-J\left(r_{0} d \chi+\sqrt{\kappa / 4 \pi} A_{\mu} d x^{\mu}\right)^{2}$

with the 5th coordinate, $\chi$, being restricted by $0<\chi$ $<2 \pi$, and the end points of this interval are identified. $A_{\mu}$ is the electromagnetic potential, and $r_{0}$ is 
an arbitrary constant. In the spherically symmetric case this give the following $5 \mathrm{D}$ metric

$$
\begin{aligned}
d s_{5}^{2}= & e^{2 \nu(r)} d t^{2}-d r^{2}-a^{2}\left(d \theta^{2}+\sin ^{2}(\theta) d \phi^{2}\right) \\
& -\left(r_{0} d \chi+\sqrt{\frac{\kappa}{4 \pi}} \omega(r) d t\right. \\
& \left.-\sqrt{\frac{\kappa}{4 \pi}} Q \cos (\theta) d \phi\right)^{2}
\end{aligned}
$$

with $J=1$ and, as before, $a=$ const. The nonvanishing components of the electromagnetic potential are

$A_{0}(r)=\omega(r), \quad A_{3}(\theta)=-\frac{Q}{a} \cos (\theta)$

The $A_{3}$ component is formally that of a magnetic monopole. With this ansatz, the 5D Kaluza-Klein field equations become [4]

$\omega^{\prime \prime}-\nu^{\prime} \omega^{\prime}=0, \quad \frac{1}{a^{2}}=\frac{\kappa}{8 \pi} \frac{Q^{2}}{a^{4}}$

$\nu^{\prime \prime}+\left(\nu^{\prime}\right)^{2}-\frac{\kappa}{8 \pi}\left(\omega^{\prime}\right)^{2} e^{-2 \nu}=0$,

$a^{4}\left(\omega^{\prime}\right)^{2}-e^{2 \nu} Q^{2}=0$

The first integral to the first equation of (12) is given by

$\omega^{\prime}=\frac{q}{a^{2}} e^{\nu}$

$q$ is a constant of integration. With Eq. (13) the last equation of (12) reads

$q^{2}=Q^{2}$

or, equivalently,

$$
\frac{\kappa}{16 \pi}\left(Q^{2}+q^{2}\right)=a^{2}
$$

Finally one can use $\omega^{\prime}$ in the third equation of Eq. (12) and also integrate it directly to obtain

$e^{\nu}=\cosh \left(\frac{r}{a}\right), \quad \omega=\frac{q}{a} \sinh \left(\frac{r}{a}\right)$

In some limited sense $Q$ can be interpreted as a magnetic charge, and $q$ as an electric charge. Both $q$ and $Q$ appear formally in the expressions of $A_{0}(r)$ and $A_{3}(\theta)$ in the same way in which charges usually appear. Further, as $q$ and $Q$ increase the strength of the electromagnetic field increases in a directly proportional manner. However, strictly there are no charges present since we are dealing with the vacuum equations, and there are no singularities in the electromagnetic potential.

This 5D solution is nearly identical to the 4D solution studied in the previous section, except in the present case we have the additional restriction $q^{2}=$ $Q^{2}$, which fixes the strengths of the "electric" and "magnetic" field to be equal as we will show next. In the 4D case one could vary the relative strengths of the electromagnetic fields (by varying $\alpha$ ). This equality between "electric" and "magnetic" fields was already noticed for other Kaluza-Klein dyon solutions [5].

As in Ref. [6] we now define the two pairs of electromagnetic 3-vectors $\boldsymbol{E}, \boldsymbol{B}$ and $\boldsymbol{D}, \boldsymbol{H}$

$E_{\alpha}=F_{0 \alpha}, \quad B^{\alpha}=-\frac{1}{2 \sqrt{\gamma}} \epsilon^{\alpha \beta \rho} F_{\beta \rho}$

and

$D^{\alpha}=-\sqrt{g_{00}} F^{0 \alpha}, \quad H_{\alpha}=-\frac{1}{2} \sqrt{\gamma} \epsilon_{\alpha \beta \mu} \sqrt{g_{00}} F^{\beta \mu}$

with $\gamma$ the determinant of the 3D metric of the $t=$ const hypersurfaces, and $\epsilon_{\alpha \beta \gamma}$ is the Levi-Civita symbol. The only non-vanishing components are

$E_{1}=-\frac{q}{a^{2}} e^{\nu}, \quad B^{1}=-\frac{q}{a^{2}}$

and

$D^{1}=-\frac{q}{a^{2}}, \quad H_{1}=-\frac{q}{a^{2}}$

The 3D electromagnetic field vectors $\boldsymbol{D}, \boldsymbol{H}, \boldsymbol{B}$ as defined above, are constant but $\boldsymbol{E}$ is not.

\section{Einstein-Yang-Mills theory}

In this section, we will examine gravity plus an SU(2) Yang-Mills field. The 4D metric that we consider is identical to that given in Eq. (4). 
For the gauge potential components $A_{\mu}^{a}$ we use the ansatz (see also Refs. [7,8]) in spherical-polar coordinates

$\left(A_{\theta}^{a}\right)=\frac{Q}{a}(\sin \phi,-\cos \phi, 0)$,

$\left(A_{\phi}^{a}\right)=\frac{Q}{a} \sin \theta(\cos \phi \cos \theta, \sin \phi \cos \theta,-\sin \theta)$,

$\left(A_{t}^{a}\right)=V(r)(\sin \theta \cos \phi, \sin \theta \sin \phi, \cos \theta)$

All other components are zero. The field strength components are defined via

$F_{\mu \nu}^{a}=A_{\nu, \mu}^{a}-A_{\mu, \nu}^{a}+g \epsilon^{a b c} A_{\mu}^{b} A_{\nu}^{c}$

with $g$ being the Yang-Mills coupling constant. Under these assumptions the gravitational part of the EYM equations reduce to

$$
\begin{aligned}
& \frac{1}{a^{2}}=\frac{\kappa e^{-2 \nu}}{8 \pi a^{4}}\left[V^{\prime 2} a^{4}+e^{2 \nu} Q^{2}(g Q-2)^{2}\right. \\
& \left.\quad-2 a^{2} V^{2}(g Q-1)^{2}\right] \\
& a^{2}\left(\nu^{\prime \prime}+\nu^{\prime 2}\right)=\frac{\kappa e^{-2 \nu}}{8 \pi a^{2}}\left[V^{\prime 2} a^{4}+e^{2 \nu} Q^{2}(g Q-2)^{2}\right]
\end{aligned}
$$

$$
\begin{aligned}
\frac{1}{a^{2}}= & \frac{\kappa e^{-2 \nu}}{8 \pi a^{4}}\left[V^{\prime 2} a^{4}+e^{2 \nu} Q^{2}(g Q-2)^{2}\right. \\
& \left.+2 a^{2} V^{2}(g Q-1)^{2}\right]
\end{aligned}
$$

The difference of (23) and (25) leads to

$Q g-1=0 \rightarrow Q=\frac{1}{g}$

Taking into account this dual restriction between $Q$ and $g$ the only non zero equation for the gauge field part of the EYM field equations is

$V^{\prime \prime}-\nu^{\prime} V^{\prime}=0$

This equation has the first integral

$V^{\prime}=\frac{q}{a^{2}} e^{\nu}$

with $q$ being a constant of integration. The third of the EYM equations, Eq. (25), together with Eq. (28) and the duality condition between $Q$ and $g$ leads to the following relation between parameters of the model and the integration constant

$$
\frac{\kappa}{8 \pi}\left(q^{2}+Q^{2}\right)=a^{2}
$$

This expression is similar to (14) we obtained for the model in the 5D Kaluza-Klein theory. Finally, a special solution of Eq. (24) is, as in the 5D KaluzaKlein theory,

$e^{\nu(r)}=\cosh \left(\frac{r}{a}\right)$.

Using this expression for $e^{\nu(r)}$ it is possible to integrate $V^{\prime}$ of Eq. (28) to obtain $V(r)$ in a form similar to $\omega(r)$ of Eq. (16). There is an important difference between the present solution and the previous Abelian one: In the latter case the integration constant $q$ has to be equal to the parameter $Q$ and $Q$ by itself is given in terms of $\kappa$ and $a^{2}$.

Based on the above solution one can put forth a rough picture of the confinement mechanism which involves the presence of gravity in a crucial way, rather than confinement being solely due to the Yang-Mills fields. At large energy scales it is more likely for "magnetic" charges to fluctuate out of the vacuum. If magnetic charge is quantized by some fundamental quanta, $Q_{0}$, then $Q=n Q_{0}$ where $n=$ $0,1,2,3 \ldots$. At larger energy scales it becomes more likely to have larger $n$ and therefore larger $Q$ fluctuate out of the vacuum. The higher $n$ values can be viewed either as $n$ magnetic charges of unit $Q_{0}$, or a single magnetic charge which carries a multiple, $n$, of the fundamental magnetic charge, $Q_{0}$. Thus as the energy scale increases $Q$ increases and $g$ decreases taking on small, perturbative values. As one lowers the energy scale the fluctuation of "magnetic" charges out of the vacuum becomes less probable so that $Q \rightarrow 0$ and $g \rightarrow \infty$. Thus in the high and low energy scale limits this EYM system can be seen as having two differing phases: For $Q$ finite and large, $g$ is small and perturbative, and one has a deconfined phase; for $Q \rightarrow 0, g$ is large and non-perturbative and one has a confined phase. Many features of the standard picture of confinement (flux tube structures, "magnetic" charges, and a gauge coupling $g$ which can become small or large) are embodied by these solutions. Unlike the standard picture where one has only Yang-Mills fields, for the present 
solutions the presence of the gravitational interaction appears to play a significant role.

Next we look at the "electromagnetic" field embedded in the non-Abelian gauge fields. As in Refs. $[8,9]$, we define the components of the "electromagnetic' U(1) gauge potential by

$A_{\mu}=n_{a} A_{\mu}^{a}$

where $\left(n_{1}, n_{2}, n_{3}\right)=(\sin \theta \cos \phi, \sin \theta \sin \phi, \cos \theta)$ is the normal vector to the unit sphere. For the ansatz given in Eq. (21) this yields

$A_{\mu}=V \delta_{\mu}^{0}$

One possible definition of an electromagnetic field tensor, which is invariant with respect to $\mathrm{SU}(2)$ transformations is

$F_{\mu \nu}=A_{\nu, \mu}-A_{\nu, \mu}-\epsilon_{a b c} n^{a} n_{, \mu}^{b} n_{, \nu}^{c}$

For the present ansatz this leads to

$F_{10}=V^{\prime}, \quad F_{23}=-\frac{Q}{a} \sin \theta$

The electromagnetic field defined this way is a solution of the 4D Einstein-Maxwell vacuum field equations. Although this form of the gauge potentials and field strength tensor is reminiscent of a magnetic monopole it possesses an additional electric component and its topological structure does not allow the localization of a source.

\section{Special vacuum equations for $D=7$}

As in Ref. [10] the multidimensional gravity theories considered in this paper are gravity on the principal bundle with either $\mathrm{U}(1)$ or $\mathrm{SU}(2)$ as the structural group. The easiest way to derive the field equations in the last case is to change over to the 7-bein formalism (with $h_{B}^{\bar{A}}$ the components of the 7-bein). The metric in this case is

$d s^{2}=\omega^{\bar{\mu}} \omega_{\bar{\mu}}+\omega^{\bar{a}} \omega_{\bar{a}}$

with

$\omega^{\bar{\mu}}=h_{\nu}^{\bar{\mu}} d x^{\nu}, \quad \omega^{\bar{a}}=\varphi\left(x^{\mu}\right)\left(r_{0} \sigma^{\bar{a}}+A_{\nu}^{\bar{a}}\left(x^{\mu}\right) d x^{\nu}\right)$

An overbar means a component with respect to an orthonormalized basis. Here, $\sigma^{\bar{a}}=h_{b}^{\bar{a}} d x^{b}$ are the
Maurer-Cartan 1-forms on the group, and $A_{v}^{\bar{a}}$ is the gauge potential. Capital letters run from 0 to 6 , lower case Greek letters from 0 to 3, and lower case Latin letters from 4 to 6 . We set $h_{b}^{\bar{\mu}}=0$ and do not vary them, so that we have only the following independent degrees of freedom: $h_{\nu}^{\bar{A}}=\left\{h_{\nu}^{\bar{\mu}} ; A_{\nu}^{\bar{a}}\right\}$ and $\varphi\left(x^{\mu}\right)$. Since the fiber of the principal bundle is a symmetric space (the SU(2) gauge group in this section) $h_{b}^{\bar{a}}$ is given and can not be varied (for the $\mathrm{SU}(2)$ gauge group they will given below). For the Lagrange density we choose the 7D curvature scalar density $h \tilde{R}\left(h=\operatorname{det}\left(h_{B}^{\bar{A}}\right) ; \tilde{R}\right.$ is the Ricci scalar). Varying with respect to only the independent degrees of freedom leads to the following gravitational field equations

$\tilde{R}_{\bar{\mu}}^{A}-\frac{1}{2} h_{\bar{\mu}}^{A} \tilde{R}=0$

and

$\left(\tilde{R}_{\bar{a}}^{b}-\frac{1}{2} h_{\bar{a}}^{b} \tilde{R}\right) h_{b}^{\bar{a}}=0$

Eq. (37), with indices $\left(\frac{a}{\mu}\right)$, are the "Yang-Mills" equations.

Now, we consider a 7D space-time with a metric which is similar to the metric of Eq. (10) (with $\varphi\left(x^{\mu}\right)=1$ in Eq. (36)) [4]

$$
\begin{aligned}
d s_{7}^{2}= & e^{2 \nu(r)} d t^{2}-d r^{2}-a^{2}\left(d \theta^{2}+\sin ^{2} \theta d \phi^{2}\right) \\
& -\sum_{a=1}^{3}\left(r_{0} \sigma^{a}-\sqrt{\frac{\kappa}{4 \pi}} A_{\mu}^{\bar{a}}(r) d x^{\mu}\right)^{2}
\end{aligned}
$$

This form of the metric is also similar to the metric of certain multidimensional cosmology models in that the metric coefficients of the internal metric depend only on the coordinates of the external 4D space-time. The length of the internal space is characterized by $r_{0}$.

Again, $a$ is a non-zero constant. The metric of the external 4D space-time is given by the first three terms in (39). The three 1-forms - $\sigma^{a}(a=1,2,3)$ are, by definition, related to the Euler angles $\alpha, \beta, \gamma$ of the SU(2) group via

$$
\begin{aligned}
\sigma^{1} & =(\sin \alpha d \beta-\sin \beta \cos \alpha d \gamma) \\
\sigma^{2} & =-(\cos \alpha d \beta+\sin \beta \sin \alpha d \gamma) \\
\sigma^{3} & =(d \alpha+\cos \beta d \gamma)
\end{aligned}
$$


The ansatz for $A_{\mu}^{\bar{a}}$ is similar to Eq. (21)

$\left(A_{\theta}^{\bar{a}}\right)=\frac{Q}{a}(\sin \phi,-\cos \phi, 0)$,

$\left(A_{\phi}^{\bar{a}}\right)=\frac{Q}{a} \sin \theta(\cos \phi \cos \theta, \sin \phi \cos \theta,-\sin \theta)$,

$\left(A_{t}^{\bar{a}}\right)=V(r)(\sin \theta \cos \phi, \sin \theta \sin \phi, \cos \theta)$

All other components are zero. The remarks made in connection with the physical and topological meaning of this ansatz are similar to the remarks made about the ansatz for the Einstein-Yang-Mills system in the previous section.

Now, let us write down the 4D Einstein-type equations following from $\tilde{R}_{1}^{1}=0$ and $\tilde{R}_{2}^{2}=0$. They read

$$
\begin{aligned}
\nu^{\prime \prime}+\left(\nu^{\prime}\right)^{2}= & \frac{\kappa}{8 \pi} e^{-2 \nu} V^{\prime 2} \\
& -\frac{\kappa}{4 \pi} V^{2}\left[2 \sqrt{\frac{\kappa}{4 \pi}} r_{0} Q-\frac{\kappa}{4 \pi} Q^{2}-r_{0}^{2}\right]
\end{aligned}
$$

$\nu^{\prime \prime}+\left(\nu^{\prime}\right)^{2}=\frac{\kappa}{8 \pi} e^{-2 \nu} V^{\prime 2}$

The difference of (44) and (45) leads to

$r_{0}=\sqrt{\frac{\kappa}{4 \pi}} Q$

With (46), the last of the 4D Einstein-type equation reads

$a^{2}-\frac{\kappa}{8 \pi} Q^{2}=0$

The 4D Yang-Mills-type equations reduce to

$V^{\prime \prime}-\nu^{\prime} V^{\prime}=0$

with the first integral

$V^{\prime}=\frac{q}{a^{2}} e^{\nu}$

where $q$ is a constant of integration. After some reformulations of the field equation $\tilde{R}_{4}^{4}+\tilde{R}_{5}^{5}+\tilde{R}_{6}^{6}=$ 0 (which is reminiscent of the 4D Klein-Gordon-type equation), we obtain

$$
\frac{\kappa}{8 \pi}\left(q^{2}+Q^{2}\right)=2 a^{2} \rightarrow q^{2}=Q^{2}
$$

Finally, again a special solution of Eq. (45) with the first integral (49) taken into account is

$e^{\nu(r)}=\cosh \left(\frac{r}{a}\right)$

Using this expression for $e^{\nu(r)}$ it is again possible to integrate $V^{\prime}$ of Eq. (49) to obtain $V(r)$ of a form similar to $\omega(r)$ of Eq. (28) This set of equations and the given solution exhibits many common points with the equations and solutions of the 5D KaluzaKlein theory and with the SU(2) EYM theory. There are some differences. For example in comparing the 5D and 7D systems one finds for the latter case that (using Eqs. (46) and (47) ) there is a relationship between the length scale of the inner and outer space, namely

$r_{0}=\sqrt{2} a$

An interesting feature of the SU(2) EYM system in particular is that the solutions with their dual relationship between $Q$ and $g$, their "magnetic charge", and their flux tube geometry mimic some of the main features of the confinement mechanism for Yang-Mills theory alone. Thus it might be conjectured that gravity plays some interesting role in the confinement mechanism. At first this conjecture appears completely unreasonable given the weak strength of the gravitational coupling compared to the gauge interaction couplings of the Standard Model. However, other recent works have explored similar ideas. First, in the large extra dimension approach [11] gravity becomes strongly coupled at an energy scale much lower than $10^{19} \mathrm{GeV}$, thus making the gravitational interaction important at these lower energy scales. Usually, however, these energy scales are greater than $1 \mathrm{TeV}$ rather than the $1 \mathrm{GeV}$ energy scale associated with the strong interaction. Second, an interesting connection between the glueball mass spectrum of pure Yang-Mills theory and supergravity was studied in Refs. [12].

\section{Concluding remarks}

The flux tube solutions studied here are significantly different (especially topologically) from the more commonly studied spherically symmetric solutions. For the flux tube solutions, the area of the 2-spheres $(r=$ const $)$ and the 2-disk does not in- 
crease with increasing "radial coordinate" $r$. Radial coordinate is put in quotes since $r$ is not a true radial coordinate since it runs from $-\infty$ to $+\infty$.

For simplicity, we only considered very special forms for the metric component $g_{00}$. Other choices of integration constants would lead to different behavior of $g_{00}$ for $r \rightarrow \infty$, for example, $g_{00} \rightarrow 0$ for $r \rightarrow \pm \infty$. There are also possible solutions which have a finite upper or lower bound for $r$. One could try to "sew" together such different solutions by matching them at some surface, $r=$ const. All these various possible solutions have the common feature that they are "cosmological" solutions - i.e. solutions that do not approach a Minkowski form. One way to make these solutions more physically useful is to view them as small scale fluctuations of some kind of space-time foam model. One easy extension of the above work would be to allow $A_{0}^{\bar{a}}$ to have imaginary components as is sometimes done in pure Yang-Mills gauge theories [7]. This would change the sign in front of the third term in equations like Eq. (12) and, the hyperbolic functions would be replaced by trigonometric functions with a finite range of $r$.

It is remarkable that different types of gravity theories admit such similar solutions. The main question concerning these flux tube solutions is their physical meaning. Maybe, they describe the ground state of certain physical systems (perhaps the interior of infinitely extended strings). Apart from the question of their physical usefulness, if any, these solutions are interesting from the topological point of view.

Finally, we summarize the following peculiarities of these solutions:

- All these solutions are functionally similar despite coming from somewhat different theories (4D Einstein gravity plus either Abelian or nonAbelian gauge fields, or Kaluza-Klein gravity). The one difference is that the Kaluza-Klein solutions fix the relative magnitudes of the electric and magnetic parts of the solutions to be equal to one another (i.e. in the 5D Kaluza-Klein case we found $q=Q$ while in the 4D Einstein-Maxwell system the magnetic and electric fields could take on any value with respect to one another). This is similar to the equality between electric and magnetic parts of the Kaluza-Klein dyons of Ref. [5].
- These gravitational solutions deserve the label flux tube spacetimes since the sections $t=$ const, $r=$ const' have constant area.

- These gravitational solutions bear some resemblance to similar flux tube structures which occur in type-II superconductors and in QCD. The SU(2) EYM solutions further have a dual relationship between the "magnetic" charge and gauge coupling, which leads to a confining/deconfining phase structure with many similarities to the standard confinement mechanism of pure Yang-Mills theory.

- In both the 4D Einstein-Yang-Mills and 7D gravity cases the gauge fields were only Abelian in nature. (i.e. even in these non-Abelian cases the fields in the flux tube were essentially "electromagnetic" fields.) This indicates some type of dynamical reduction of the gauge group: $\mathrm{SU}(2)$ $\rightarrow \mathrm{U}(1)$ in the flux tube. This leads to the possible hypothesis that any gravitational flux tube could only be filled with a U(1) gauge field.

- These properties of the flux tube spacetimes can be summarized graphically:
(4D Einstein-Yang-Mills) $\leftrightarrow$
(7D gravity)
$\downarrow$

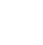
(4D Einstein-Maxwell) $\quad \leftrightarrow \quad$ (5D Kaluza-Klein)

The vertical arrows indicate a dynamical reduction $\mathrm{SU}(2) \rightarrow \mathrm{U}(1)$ and the horizontal arrows indicate a similarity of the corresponding solutions.

\section{Acknowledgements}

V.D. is grateful for financial support by a Georg Forster Research Fellowship from the Alexander von Humboldt Foundation and H.-J. Schmidt for invitation to Potsdam University.

\section{References}

[1] T. Levi-Civita, Atti Acad. Naz. Lincei 26 (1917) 519.

[2] B. Bertotti, Phys. Rev. 116 (1959) 1331; I. Robinson, Bull. Akad. Pol. 7 (1959) 351.

[3] E.I. Guendelman, Gen. Rel. Grav. 23 (1991) 1415.

[4] V.D. Dzhunushaliev, D. Singleton, Class. Quant. Grav. 16 (1999) 973. 
[5] M.J. Perry, Phys. Lett. B 137 (1984) 171.

[6] L.D. Landau, E.M. Lifschitz, Klassische Feldtheorie., Akademie-Verlag Berlin, 1984.

[7] J.P. Hsu, E. Mac, J. Math. Phys. 18 (1977) 100.

[8] G. 't Hooft, Nucl. Phys. B 79 (1974) 276.

[9] J.P. Arafune, G.O. Freund, C.J. Goebel, J. Math. Phys. 16 (1975) 433.
[10] V.D. Dzhunushaliev, Gen. Rel. Grav. 30 (1998) 583.

[11] I. Antoniadis et al., Phys. Lett. B 436 (1998) 257; N. Arkani-Hamed, S. Dimopoulos, G. Dvali, Phys. Rev. D 59 (1999) 086004.

[12] C. Csaki et al., JHEP 9901 (1999) 017; R.M. Koch et al., Phys. Rev. D 58 (1998) 105009. 